Original

\title{
Adaptación cultural y validación de la versión española de la Escala de Autoconcepto en BRCA en portadoras con alto riesgo de cáncer de mama y ovario hereditario
}

\author{
Vanessa Castejón ${ }^{a, b}$, Tatiana Rovira $^{\mathrm{b}}$, Enric C. Sumalla ${ }^{\mathrm{a}}$, Esther Darder ${ }^{\mathrm{c}}$, Silvia Iglesias ${ }^{\mathrm{a}}$, \\ Cristian Ochoa ${ }^{\mathrm{d}, \mathrm{e}}$ e Ignacio Blanco ${ }^{\mathrm{a}, \mathrm{f}, *}$

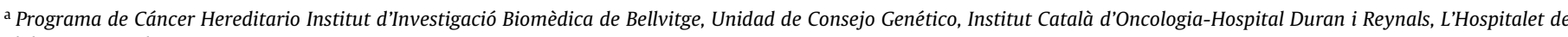 \\ Llobregat, Barcelona, España

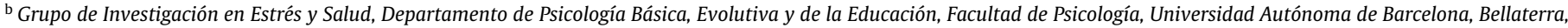 \\ Barcelona, España

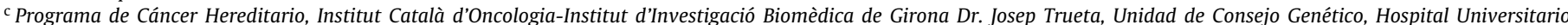 \\ Dr. Josep Trueta, Girona, España \\ d Unidad de Psico-Oncología, Hospital Duran i Reynals, L'Hospitalet de Llobregat, Barcelona, España \\ e Departamento de Psiquiatría y Psicobiología Clínica, Universidad de Barcelona, Barcelona, España \\ ${ }^{\mathrm{f}}$ Programa de Asesoramiento y Genética Clínica, Hospital Universitario Germans Trias i Pujol, Badalona, Barcelona, España
}

\section{INFORMACIÓN DEL ARTÍCULO}

\section{Historia del artículo:}

Recibido el 28 de mayo de 2015

Aceptado el 3 de septiembre de 2015

On-line el xxx

Palabras clave:

Autoconcepto

Cáncer de mama y ovario hereditario

Adaptación psicológica

Estudio de validación

\begin{abstract}
R E S U M E N
Fundamento y objetivo: Tener una predisposición hereditaria al cáncer puede asociarse a un impacto psicológico. Uno de los objetivos del consejo genético es favorecer la adaptación psicológica a la nueva situación, siendo necesarios instrumentos validados en este contexto. Por ser el autoconcepto un buen indicador de adaptación a la enfermedad o al riesgo de tenerla, y una variable relevante en oncología, el objetivo es adaptar culturalmente al castellano y validar la BRCA Self-Concept Scale.

Material y método: Ciento sesenta y cinco portadoras de mutación en los genes BRCA respondieron al cuestionario previamente sometido a un proceso de traducción-retrotraducción, y a la Escala de Preocupación por el Cáncer (EPC) como medida de validez convergente. Se valoró la estructura del cuestionario mediante una prueba de expertos y un análisis factorial confirmatorio (AFC), se calculó el $\alpha$ de Cronbach de las 3 subescalas (Estigma, Vulnerabilidad y Control), y se correlacionaron con la EPC.

Resultados: La prueba de expertos y el AFC no confirman la estructura original del cuestionario. El modelo reespecificado (con los ítems 10 y 13 en Vulnerabilidad) muestra mejor ajuste: comparative fit index 0,973; Tucker-Lewis index 0,968; root mean square error of approximation 0,067. El $\alpha$ de Cronbach es de 0,83 para Estigma, de 0,84 para Vulnerabilidad, y de 0,61 para Control. Se encuentra evidencia de validez convergente con la EPC (rho de Spearman 0,631 para Estigma, 0,683 para Vulnerabilidad, y -0,363 para Control; $\mathrm{p}<0,001$ ).

Conclusiones: Los resultados apoyan la validez de la escala modificada de autoconcepto BRCA, siendo una medida potencialmente útil para valorar la adaptación a tener una alta predisposición hereditaria al cáncer.
\end{abstract}

(C) 2015 Elsevier España, S.L.U. Todos los derechos reservados.

\section{Cultural scale adaptation and validation of the Spanish version of the BRCA Self-Concept Scale in women carriers at high risk for hereditary breast and ovarian cancer}

\section{A B S T R A C T}

Background and objective: Having an inherited predisposition to cancer may have a psychological impact, and one goal of genetic counseling is to promote psychological adjustment to the new situation. Thus, in the genetic context, validated measures of adjustment are required. Given that self-concept is a good

Self-concept

Hereditary breast and ovarian cancer syndrome

\footnotetext{
* Autor para correspondencia.

Correo electrónico: iblanco.germanstrias@gencat.cat (I. Blanco).
} 
Psychological adjustment Validation indicator of adjustment to the disease or to the risk for it, and a relevant variable in oncology, the goal of the study is to culturally adapt and validate the BRCA Self-Concept Scale.

Material and Method: One hundred and sixty-five BRCA carriers' women answered to the questionnaire, previously adapted through a process of forward/back-translation, and to the Cancer Worry Scale (CWS) as a measure of convergent validity. Theoretical structure of BRCA Self-Concept Scale was assessed by expert judges, and submitted to a confirmatory factor analysis (CFA). Cronbach's $\alpha$ was calculated for each subscale (Stigma, Vulnerability and Control), and correlations with CWS were performed.

Results: Expert judges' structure and CFA do not support the original structure of the questionnaire. The respecificity model (with items 10 and 13 loading on Vulnerability factor) show a better fit: comparative fit index 0.973; Tucker-Lewis index 0.968; root mean square error of approximation 0.067. The Cronbach's $\alpha$ is 0.83 for Stigma, 0.84 for Vulnerability, and 0.61 for Control. Evidence of convergent validity with CWS has been obtained (Spearman's rho 0.631 for Stigma, 0.683 for Vulnerability, and -0.363 for Control; $P<.001)$.

Conclusions: Results support the validity of the modified Spanish BRCA Self-Concept Scale, which is a potentially useful measure for the study of psychological adjustment to high risk for hereditary breast and ovarian cancer.

(c) 2015 Elsevier España, S.L.U. All rights reserved.

\section{Introducción}

Entre un 5 y un $10 \%$ de los diagnósticos de cáncer de mama (CM) se deben a mutaciones en la línea germinal de los genes de alta penetrancia BRCA1/2 que se transmiten con un patrón de herencia autosómico dominante, responsables del síndrome de cáncer de mama y ovario hereditario $(\mathrm{CMOH})$. Las portadoras de mutaciones en estos genes tienen un alto riesgo acumulado de desarrollar $\mathrm{CMOH}$ a lo largo de la vida, que oscila entre el 47-52\% para el CM y el $18-22 \%$ para el cáncer de ovario $(\mathrm{CO})^{1}$, y una probabilidad del $50 \%$ de transmitir esta susceptibilidad a su descendencia. Ser portador de una condición genética que predispone a una enfermedad potencialmente mortal como el cáncer plantea una amenaza a la propia salud, y puede considerarse un suceso vital altamente estresante ${ }^{2}$. El conocimiento de este riesgo incrementado es para toda la vida, y afecta no solo al individuo, sino a su familia, que sobre la base de una información probabilística deberá hacer frente a una serie de decisiones complejas. Los individuos y familias susceptibles de presentar esta predisposición hereditaria al cáncer son derivados a las unidades de consejo genético (CG), donde se realiza una valoración individualizada del riesgo de ocurrencia y/o recurrencia de la enfermedad, y se ofrecen estrategias de prevención y detección precoz, acordes al riesgo individual, que hayan demostrado efectividad en disminuir las tasas de incidencia y mortalidad. El objetivo del CG es ayudarles a conocer y a adaptarse a las implicaciones médicas, psicológicas y familiares de la contribución genética a la enfermedad ${ }^{3}$.

Favorecer la adaptación psicológica a la condición de portador es uno de los objetivos del CG, y es importante poder evaluarla. La adaptación a una enfermedad hereditaria o al riesgo de tenerla es dinámica y multidimensional, y son necesarios indicadores tanto de proceso como de resultado para demostrar la efectividad del $\mathrm{CG}^{4-6}$. Para valorar la adaptación psicológica en el contexto del CG se utilizan casi en exclusiva cuestionarios estandarizados de síntomas de ansiedad y depresión, que son medidas del fracaso en la adaptación -resultado-, y no medidas observables de cómo se está desarrollando el proceso. Así mismo, se ha advertido de la falta de sensibilidad de estos cuestionarios a necesidades específicas y relevantes del $\mathrm{CG}^{2,4,7}$. En este sentido, un estudio con mujeres diagnosticadas de CM o CO que se realizaron el test genético constató que el deseo de recibir soporte psicológico responde a otras necesidades no detectadas por los indicadores habituales focalizados en la psicopatología, y que solo un tercio de quienes expresan estas necesidades reciben finalmente ayuda psicológica ${ }^{8}$. En definitiva, son necesarios instrumentos sensibles a las necesidades específicas del CG, adecuadamente validados en este contexto, y que sean medidas multidimensionales que den cuenta de los aspectos relevantes del proceso de adaptación a enfermedades hereditarias ${ }^{2,4-7}$.

En este contexto se ha desarrollado y validado en lengua inglesa la BRCA Self-Concept Scale ${ }^{2}$, que evalúa el impacto en el autoconcepto de saberse portador de una alteración genética predisponente al $\mathrm{CMOH}$, y tiene sus equivalentes en otros síndromes oncológicos hereditarios ${ }^{9,10}$. El autoconcepto, basado en el Modelo de Esquemas del Autoconcepto, se define como una estructura cognitiva compleja, multidimensional, activa y dinámica, que integra y sintetiza pensamientos, sentimientos y experiencias sobre uno mismo ${ }^{11}, y$ es considerado el marco organizador que dota de sentido, forma y dirección a estas experiencias ${ }^{12}$. En enfermedades crónicas, un autoconcepto nuevo o restaurado es indicativo de adaptación a la condición o enfermedad en tanto que refleja su aceptación afectiva e internalización de uno mismo como persona con una determinada condición ${ }^{13}$. En oncología el autoconcepto es una variable de relevancia, ya que su alteración puede comportar mayor malestar emocional e impactar negativamente en la adherencia a las conductas de salud. Alternativamente, la habilidad para construir nuevos esquemas sobre uno mismo puede facilitar la recuperación tras un episodio adverso, estableciéndose así una relación bidireccional autoconcepto-enfermedad ${ }^{14}$.

La BRCA Self-Concept Scale evalúa el impacto de ser portador en las diferentes dimensiones del autoconcepto, considerando tanto cambios negativos como positivos. El análisis de componentes principales realizado para su desarrollo y validación arroja una solución de 3 factores que dan muestra de su multidimensionalidad: Estigma, que en la versión original representa el 38,7\% de la variancia total explicada ( $\alpha$ de Cronbach $=0,86$ ), evalúa hasta qué punto la persona se siente aislada, diferente a los otros, o con una identidad dañada tras someterse al test genético; Vulnerabilidad (8,5\% de la variancia total explicada en la versión original, $\alpha$ de 0,76 ) evalúa la fragilidad e incertidumbre respecto al propio cuerpo y la salud; Control (6,5\% de la variancia total explicada en la versión original, $\alpha$ de 0,68 ) evalúa la autoeficacia, la resiliencia y la esperanza ${ }^{2}$. Los estudios realizados hasta la fecha no han reportado la existencia de un factor de segundo orden, centrándose la atención en la afectación de las dimensiones específicas del autoconcepto y su asociación con otras variables relevantes del CG. Así, integrar la condición de alto riesgo a desarrollar $\mathrm{CMOH}$ en el autoconcepto en términos de Control se asocia a menores niveles de malestar emocional; sin embargo, hacerlo en términos de Estigma y/o Vulnerabilidad se asocia a niveles más altos. Así mismo, se ha demostrado que una comunicación abierta con la pareja e hijos se asocia a menor Estigma en el autoconcepto, lo que revierte en menores niveles de malestar general ${ }^{15-17}$. 
Este estudio tiene como objetivo adaptar culturalmente la BRCA Self-Concept Scale al castellano, confirmar su estructura factorial original, evaluar su consistencia interna y analizar su convergencia con la Escala de Preocupación por el Cáncer (EPC), único instrumento validado al castellano en el contexto de CM hereditario ${ }^{18}$ y que ha mostrado relación con la adherencia a las conductas preventivas ${ }^{19}$.

\section{Material y método}

\section{Participantes}

Para obtener una muestra similar a la del instrumento original, se identificaron mujeres portadoras de mutaciones patogénicas en los genes BRCA1/2, tanto sanas como afectadas, en seguimiento médico dado su alto riesgo de padecer $\mathrm{CM}$ y $\mathrm{CO}$. Son criterios de inclusión: ser portadora de mutación en BRCA1/2, ser mayor de edad, saber leer y escribir castellano, y que hubieran transcurrido 6 meses como mínimo desde la entrega del resultado del test genético. Son criterios de exclusión: tener un deterioro cognitivo y/o un diagnóstico psiquiátrico incapacitante. De las 329 candidatas invitadas a participar, 165 (50,15\%) devolvieron los cuestionarios completados, sin diferencias significativas de edad ni en las variables médicas entre las que aceptan participar y las que rechazan (datos no reportados, disponibles si se solicitan). El tamaño final de la muestra cumple los criterios ratio sujeto:ítem 5:1-10:1, adecuado para aplicar un análisis factorial confirmatorio (AFC) ${ }^{20}$. La edad promedio fue de 47,73 años ( $\mathrm{DE}=13,38)$, y $76(46,1 \%)$ no habían desarrollado ningún cáncer en el momento de contestar el cuestionario, siendo consideradas portadoras sanas. Los descriptivos de la muestra final se muestran en la tabla 1.

\section{Instrumentos de medida}

BRCA Self-Concept Scale ${ }^{2}$ : evalúa el impacto en el autoconcepto de ser portadora de mutación en BRCA1/2. Está formada por 17 ítems con un formato de respuesta tipo Likert con 8 categorías, en un rango de puntuación de uno (muy en desacuerdo) a 7 (muy de acuerdo) en sentido creciente. La puntuación 8 corresponde a la categoría no aplicable (NA). El factor Estigma contiene los ítems $5,8,9,10,12,13,16$ y 17 . El factor Vulnerabilidad comprende los ítems 3, 4, 7, 11 y 14. El factor Control incluye los ítems 1, 2, 6 y 15. Mayores puntuaciones en Estigma y Vulnerabilidad implican cambios negativos en el autoconcepto; en Control, implican cambios positivos.

$E P C^{18}$ : autoinforme de 6 ítems que evalúa la preocupación por desarrollar cáncer, con un rango de puntuación que oscila de uno (ninguna preocupación) a 4 (máxima preocupación); $\alpha$ en el presente estudio de 0,896 .

Variables sociodemográficas: edad, nivel de estudios, estado civil, hijos.

Variables médicas: estatus oncológico, tiempo desde el diagnóstico genético, tiempo desde el diagnóstico oncológico y cirugías profilácticas.

\section{Recogida de datos}

El estudio se ha realizado en las unidades de CG del Institut Català d'Oncologia ubicadas en los hospitales Duran i Reynals, Germans Trias i Pujol y Josep Trueta, previa revisión y aprobación de los comités éticos de investigación clínica. La muestra se obtuvo entre julio de 2012 y mayo de 2014, siguiendo los protocolos establecidos para el acceso y el tratamiento de los datos de las historias
Tabla 1

Características generales de la muestra $(n=165)$

\begin{tabular}{lc}
\hline Hospital de procedencia, $n$ (\%) & \\
$\quad$ Duran i Reynals & $95(57,6)$ \\
Germans Trias i Pujol & $22(13,3)$ \\
Josep Trueta & $48(29,1)$ \\
Edad en años, media (DE) & $47,73(13,38)$ \\
Estudios completados, $n$ (\%) & \\
Sin estudios & $4(2,4)$ \\
Primarios & $65(39,4)$ \\
Medios & $56(33,9)$ \\
Superiores & $40(24,2)$ \\
Estado civil, $n$ (\%) & \\
Soltera & $17(10,3)$ \\
Casada/conviviendo & $129(78,2)$ \\
Separada & $12(7,3)$ \\
Viuda & $7(4,2)$ \\
Tiene hijos, $n$ (\%) & $130(78,8)$ \\
Meses desde el diagnóstico genético, media (DE) & $47,02(37,11)$ \\
Estatus oncológico, $n$ (\%) & \\
Sana & \\
Afectada & $76(46,1)$ \\
Primer diagnóstico oncológico de las afectadas (n=89), $n(\%)$ & $89(53,9)$ \\
Mama & \\
Ovario & $75(84,3)$ \\
Otros & $12(13,5)$ \\
Años desde el primer diagnóstico oncológico, media (DE) & $2(2,2)$ \\
Cirugía profiláctica, $n$ (\%) & $9,55(8,71)$ \\
$\quad$ Ninguna & \\
Mastectomía bilateral & \\
Salpingooforectomía & \\
Mastectomía bilateral + Salpingooforectomía & \\
& \\
\hline & \\
\hline
\end{tabular}

clínicas y garantizando la confidencialidad de estos. Se envió por correo postal una explicación del estudio con los cuestionarios, el consentimiento informado y un sobre con franqueo pagado. Todos los participantes fueron informados de la confidencialidad de sus respuestas y aceptaron contestar voluntariamente a los cuestionarios.

\section{Adaptación de la escala}

La autora autorizó la adaptación cultural y validación de la escala al castellano, que se desarrolló mediante 2 procedimientos complementarios: traducción-retrotraducción, siguiendo las directrices de la Comisión Internacional de Tests ${ }^{21}$, y prueba de expertos para acumular evidencias de validez de contenido de los ítems traducidos en relación con su adscripción a las subescalas del cuestionario. La versión inglesa del instrumento fue traducida por 2 personas bilingües (una lingüista y una psicóloga), y retrotraducida por otras 2 personas con el mismo perfil, todas ellas conocedoras de ambas culturas. Las 2 traducciones en castellano fueron revisadas por el equipo de investigación y las traductoras, generándose una primera versión de la Escala de Autoconcepto en $B R C A$, que se envió posteriormente a retrotraducir al inglés. La versión de la escala resultante se evaluó en los niveles de equivalencia recomendados y se consensuó que nuestra versión era fiel a la original. Finalmente, se evalúo la claridad, la comprensión, el tiempo de cumplimentación, la relevancia y la aceptabilidad mediante la administración piloto de la escala a una submuestra de 10 pacientes. Para la prueba de expertos, 5 psicólogos de la salud valoraron en una escala dicotómica "sí/no» el nivel de adecuación de cada ítem para cada subescala, teniendo en cuenta la definición de la autora. Además, debían indicar el grado de seguridad de cada juicio emitido, en una escala de 0 a 100. Para valorar la adecuación de cada ítem a su subescala se dividió el juicio de seguridad por 100, y se multiplicó el resultado por 
Tabla 2

Descriptivos de los ítems de la Escala de Autoconcepto en $B R C A(\mathrm{n}=165)$

\begin{tabular}{|c|c|c|c|c|c|c|c|c|c|}
\hline Ít._Factor original & Media & $\mathrm{DE}$ & Asimetría ET = 0,192 & Curtosis ET = 0,383 & Efecto suelo, \% & Efecto techo, \% & NA & $\mathrm{NC}$ & perdidos $(\mathrm{NA}+\mathrm{NC}), \%$ \\
\hline 1: esperanzado/a_C & 5,48 & 1,471 & $-0,899$ & 0,030 & 0,6 & 28,80 & 3 & 2 & 3,03 \\
\hline 2: capaz manejar resultado_C & 5,61 & 1,615 & $-1,347$ & 1,181 & 4,40 & 36,70 & 6 & 1 & 4,24 \\
\hline 3: traicionado/a por mi cuerpo_V & 2,93 & 2,020 & 0,673 & $-0,894$ & 36,80 & 7,70 & 8 & 2 & 6,06 \\
\hline 4: bomba de relojería_V & 2,48 & 1,913 & 0,972 & $-0,488$ & 50,90 & 3,70 & 2 & 0 & 1,21 \\
\hline 5: diferente a mi edad_E & 2,84 & 2,204 & 0,753 & $-0,993$ & 48,10 & 10,50 & 2 & 1 & 1,82 \\
\hline 6: conozco bien mi cuerpo_C & 5,33 & 1,639 & $-1,069$ & 0,447 & 4,30 & 26,70 & 1 & 3 & 2,42 \\
\hline 7: culpable transmisión hijos_V & 4,19 & 2,346 & $-0,188$ & $-1,513$ & 24,20 & 26,10 & 6 & 2 & 4,85 \\
\hline 8: aislado/a_E & 1,53 & 1,110 & 2,632 & 7,241 & 72,50 & 0,60 & 4 & 1 & 3,03 \\
\hline 9: pérdida intimidad_E & 2,21 & 1,782 & 1,343 & 0,533 & 56,30 & 3,80 & 4 & 1 & 3,03 \\
\hline 10: pensar en el resultado_E & 3,11 & 1,892 & 0,448 & $-1,107$ & 26,70 & 4,30 & 2 & 0 & 1,21 \\
\hline 11: preocupación cáncer_V & 5,09 & 2,081 & $-0,915$ & $-0,499$ & 12,50 & 35,00 & 5 & 0 & 3,03 \\
\hline 12: etiquetado/a EE & 2,59 & 2,060 & 0,999 & $-0,419$ & 50,90 & 8,80 & 6 & 0 & 3,64 \\
\hline 13: agobiado/a_E & 2,46 & 1,740 & 0,935 & $-0,332$ & 45,60 & 2,50 & 6 & 1 & 4,24 \\
\hline 14: desconfío de mi cuerpo_V & 2,72 & 1,927 & 0,797 & $-0,648$ & 41,70 & 5,80 & 6 & 3 & 5,45 \\
\hline 15: tengo el control_C & 3,91 & 1,897 & $-0,081$ & $-1,061$ & 15,80 & 9,50 & 5 & 2 & 4,24 \\
\hline 16: afecta a quien soy_E & 2,35 & 1,766 & 1,156 & 0,189 & 50,00 & 3,80 & 6 & 3 & 5,45 \\
\hline 17: reservado/a_E & 2,25 & 1,793 & 1,460 & 0,934 & 50,3 & 5,1 & 7 & 1 & 4,85 \\
\hline
\end{tabular}

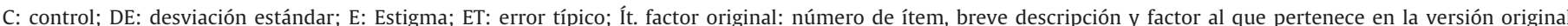
(descripción completa en la fig. 1); NA: no aplicable; NC: no contestado; V: vulnerabilidad.

-1 cuando la respuesta en la escala dicotómica era «no» (resultado de juzgar el ítem como no perteneciente a esa subescala). Para cada ítem se obtuvieron 3 puntuaciones, una por subescala, de $-1 \mathrm{a}+1$. Para cada uno de estos valores resultantes se realizó una prueba no paramétrica de contraste de hipótesis (Kolmogorov-Smirnov), con valor de prueba de 0 , para determinar su significación, usando un criterio corregido a 3 comparaciones por ítem de $\mathrm{p}<0,017$. Los resultados indican que únicamente los ítems 10 («Pienso mucho en el resultado de mi prueba genética»), y 13 («Me siento agobiada con esta información»), considerados de Estigma en el instrumento original, no muestran pertenencia a su subescala $\left(z_{k-s}=0,80\right.$, ns; y $\mathrm{z}_{\mathrm{k}-\mathrm{s}}=0,87$, ns, respectivamente), y sí lo hacen, según los jueces, a la escala de Vulnerabilidad $\left(z_{k-s}=1,70 ; p=0,006\right.$ y $z_{k-s}=1,62 ; p=0,01$, respectivamente). Por ello se decidió poner a prueba, además del modelo original de la autora (modelo 1 ), el modelo en el que los ítems 10 y 13 pasan a formar parte de la subescala de Vulnerabilidad (modelo 2).

\section{Análisis}

Para evaluar las propiedades psicométricas de los 2 modelos sometidos a prueba se realizó: a) análisis descriptivo de los ítems; b) AFC mediante el estimador de mínimos cuadrados ponderados firmes para datos categóricos, y sin imputación de valores perdidos por presentar estos patrones aleatorios que permiten descartar tendencias de respuesta ${ }^{22}$; c) análisis de la consistencia interna de las subescalas de ambos modelos, mediante el coeficiente $\alpha$ de Cronbach, y d) evidencia de validez convergente de las subescalas de ambos modelos con una medida equivalente de adaptación psicológica en el contexto del CG (EPC), mediante la correlación de Spearman, para confirmar las relaciones positivas y moderadas con las subescalas de Estigma y Vulnerabilidad, y negativa y moderada con la de Control.

En el AFC, además de poner a prueba el modelo 1 original, se pone a prueba un modelo reespecificado con las covariancias de error (uniqueness) de los ítems 4 («Me siento como una bomba de relojería andante») y 5 («Me siento diferente a la gente de mi edad") por ser correlativos, empezar con las mismas palabras y presentar índices de modificación (IM) que avalan este ajuste ${ }^{23}$. Esta reespecificación se mantiene en el caso del modelo 2. La bondad de ajuste de los 3 modelos se evalúa mediante los índices habituales, $\chi^{2}$, comparative fit index (CFI), Tucker-Lewis index (TLI), root mean square error of approximation (RMSEA) y weighted root mean square residual, considerando los siguientes puntos de corte: RMSEA $<0,05$ y TLI y CFI $>0,95$ como indicadores de un buen ajuste, y RMSEA $<0,08$ y TLI y CFI $>0,90$ como ajustes razonables ${ }^{20,24}$. La consistencia interna es adecuada a partir de un $\alpha$ de Cronbach de por lo menos $0,70^{25}$. Para la validez convergente, debido al tamaño medio de la muestra, una $|R| \geq 0,030$ se considera un buen tamaño del efecto ${ }^{26}$. El AFC se ha realizado con Mplus v. 7, y los demás, con SPSS v. 21.

\section{Resultados}

Los estadísticos descriptivos de los ítems se muestran en la tabla 2. Todos los ítems correspondientes a la subescala Estigma de la versión original muestran un marcado efecto suelo, sin efecto techo, dando lugar a distribuciones asimétricas positivas. Los ítems 3, 4 y 14 de Vulnerabilidad presentan también una baja representación de las categorías de mayor puntuación, mientras que los ítems 7 y 11 presentan índices de asimetría en la dirección opuesta al resto de los ítems de la subescala. Los ítems de la escala Control, cuyo contenido semántico es de valencia positiva respecto al resto de los ítems de la escala, presentan una asimetría negativa. Debido a la distribución de los ítems, los datos se analizan como categóricos. Hay un total de 102 valores perdidos (el 3,64\% del total de los valores de la muestra). El 77,45\% de los datos faltantes corresponden a la categoría NA, y 22,55\% son valores no contestados (NC). Para todos los ítems, tanto el número de NC por un lado, como el de NA por otro, representan valores inferiores al $5 \%$ de los sujetos que no contestan, y considerados conjuntamente solo los ítems 3, 14 y 16 superan ligeramente el $5 \%$, de los cuales la mayoría se corresponden con NA. Al analizar el conjunto de valores perdidos se encuentran hasta 36 patrones diferentes.

La tabla 3 muestra los índices de ajuste para los modelos analizados. El modelo 1, estructura original de 3 factores de primer orden, obtiene unos índices de ajuste que no satisfacen plenamente los estándares recomendados, especialmente para el valor RMSEA 0,083 (IC 90\% 0,068-0,097). La correlación entre Estigma y Vulnerabilidad es de 0,952 , indicando un solapamiento entre ambos factores. El modelo 1 reespecificado (liberando covariancia de error de los ítems 4 y 5), menos restrictivo, obtiene índices de ajuste más adecuados (CFI 0,969, TLI 0,963 y RMSEA 0,072 [IC 90\% 0,0570,087]), si bien mantiene una alta correlación entre los factores Estigma y Vulnerabilidad de 0,929 . Se pone a prueba el modelo 2 , en el que de acuerdo con los resultados de la prueba de expertos, los ítems 10 y 13 pasan a ser indicadores de Vulnerabilidad y en el que se mantiene liberado el parámetro de la covariancia de errores entre 


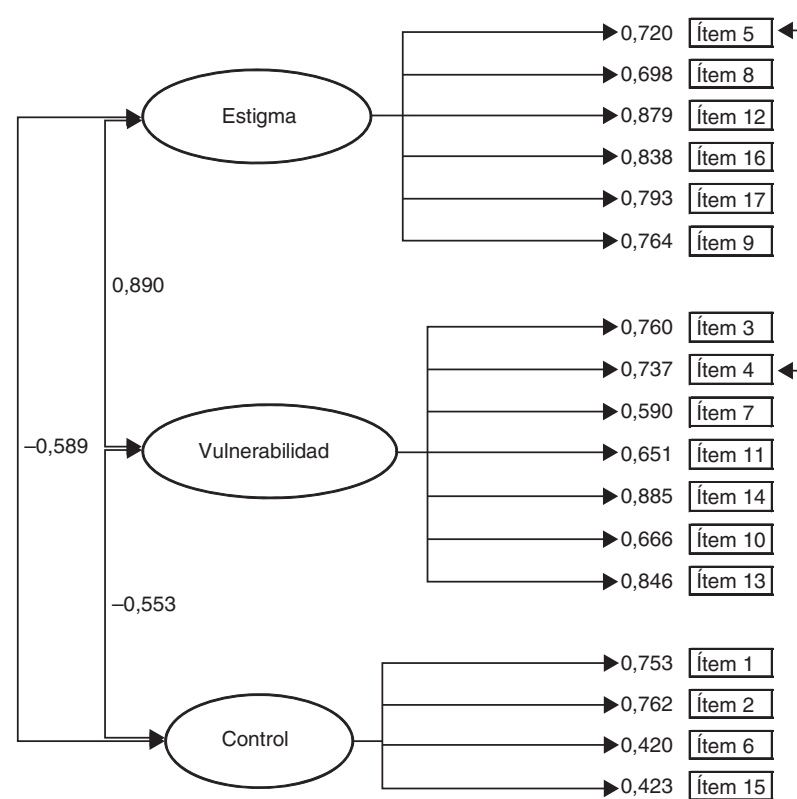

\author{
Me siento diferente a la gente de mi edad \\ Me siento aislado/a a causa del resultado de mi prueba genética \\ Me siento etiquetado/a \\ El resultado de mi prueba genética afecta a quién soy en realidad \\ Me he vuelto más reservado/a \\ Siento que he perdido mi sensación de intimidad
}

Siento que mi cuerpo me ha traicionado

Me siento como una bomba de relojería andante

Me siento culpable porque podría transmitir el riesgo de padecer cáncer a mis hijos

Me preocupa que detecten un cáncer cuando voy a los controles

Desconfío de mi cuerpo

Pienso mucho en el resultado de mi prueba genética

Me siento agobiado con esta información

Me siento esperanzado/a sobre mi propio futuro

Soy capaz de manejar el resultado de mi prueba genética

Conozco bien mi cuerpo

Tengo el control de mi salud

Parámetros estandarizados: pesos factoriales y correlaciones entre factores $(p<0,05)$
Varianzas y covarianzas residuales omitidas

Figura 1. Análisis factorial confirmatorio de 3 factores de primer orden de la versión castellana de la Escala de Autoconcepto en BRCA (modelo 2).

los ítems 4 y $5\left(\varepsilon_{45}\right)$. El modelo 2 (los ítems 10 y 13 son indicadores de Vulnerabilidad, y liberando el parámetro $\varepsilon_{45}$ ) obtiene índices de ajuste adecuados: CFI 0,973; TLI 0,968; RMSEA 0,067 (IC 90\% 0,051-0,082). Los ítems fueron buenos indicadores de los factores latentes, con cargas factoriales estandarizadas, todas significativas (entre 0,698 y 0,879 para Estigma, 0,590 y 0,885 para Vulnerabilidad, y 0,420 y 0,762 para Control). Las correlaciones entre factores, todas significativas y de signo acorde al contenido de las subescalas, fueron de 0,89 entre Vulnerabilidad y Estigma, -0,589 entre Control y Estigma, y -0,553 entre Control y Vulnerabilidad.

La consistencia interna de la escalas del autoconcepto BRCA y su correlación con la EPC pueden verse en la tabla 4. La fiabilidad de Estigma está soportada en ambos modelos con valores de 0,859 y 0,835 ; y Vulnerabilidad pasa de un valor de 0,788 en el modelo 1 a 0,843 en el modelo 2 , mientras que la escala control obtiene un valor de 0,614. Finalmente, la eliminación de cualquiera de los ítems no reportó mejora alguna en la consistencia interna de las subescalas, por lo que se decide mantenerlas íntegras. En cuanto a la correlación convergente del autoconcepto BRCA (modelo2) con la EPC, Estigma y Vulnerabilidad muestran una asociación significativa positiva alta con EPC, con valores de 0,631 y 0,683, respectivamente, mientras que Control muestra una asociación significativa negativa moderada de 0,363 .

Tabla 3

Índices de ajuste de los modelos puestos a prueba en el análisis factorial confirmatorio con estimador de mínimos cuadrados ponderados firmes para datos categóricos

\begin{tabular}{llllll}
\hline Modelos & $\chi^{2}$ & $d f$ & CFI & TLI & RMSEA (IC 90\%) \\
\hline Modelo 1 & 246,55 & 116 & 0,959 & 0,952 & $0,083(0,068-0,097)$ \\
$\begin{array}{c}\text { Modelo 1 liberando } \\
\quad \text { parámetro it4-5 }\end{array}$ & 213,96 & 115 & 0,969 & 0,963 & $0,072(0,057-0,087)$ \\
Modelo 2 & 200,008 & 115 & 0,973 & 0,968 & $0,067(0,051-0,082)$ \\
\hline
\end{tabular}

CFI: comparative fit index; IC: intervalo de confianza; Modelo 1: 3 factores de primer orden, versión original; Modelo 1 liberando parámetro it4-5: 3 factores de la versión original, liberando covariancia error ítems 4 y 5; Modelo 2: jueces, con ítems 10 y 13 como indicadores de vulnerabilidad, y covariancia error ítems 4 y 5 liberada; RMSEA: root mean square error of approximation; TLI: Tucker-Lewis index.

\section{Discusión}

Uno de los objetivos del CG en $\mathrm{CMOH}$ es facilitar la adaptación a tener un riesgo significativamente superior al resto de la población general de desarrollar la enfermedad, así como de transmitir esta susceptibilidad al cáncer a la descendencia ${ }^{3}$. Cómo los portadores de una predisposición hereditaria integran su condición de personas con alto riesgo a desarrollar cáncer en su autoconcepto es indicativo de su adaptación psicológica a este ${ }^{13,14}$. Disponer de una escala que evalúe el impacto de ser portador en el autoconcepto da respuesta a la necesidad de disponer de instrumentos sensibles y específicos adecuadamente validados en el contexto del $\mathrm{CG}^{5,6}$. Este estudio es el primero en adaptar y validar en nuestro entorno sociocultural la Escala de Autoconcepto en $B R C A$, que, frente al síntoma psicopatológico, se ha mostrado sensible y específico a las necesidades relevantes del $\mathrm{CG}^{2}$, y se erige como elemento integrador tanto del proceso como del resultado de la adaptación al estatus de portador o alto riesgo ${ }^{2,13,14}$. Para la adaptación transcultural y la validación se ha seguido un proceso protocolizado de traducción-retrotraducción, complementado con una prueba de expertos, y es el primer estudio en comprobar la estructura de la escala mediante un AFC. Los resultados del modelo 2 permiten mantener la solución de 3 factores de primer orden, Estigma, Vulnerabilidad y Control, con unos adecuados índices de ajuste. Además,

\section{Tabla 4}

Fiabilidad de las subescalas de Autoconcepto en BRCA y convergencia con Escala de Preocupación por el Cáncer

\begin{tabular}{lllr}
\hline & Estigma & Vulnerabilidad & Control \\
\hline$\alpha$ modelo 1 & 0,859 & 0,788 & 0,614 \\
$\alpha$ modelo 2 & 0,835 & 0,843 & 0,614 \\
$\begin{array}{l}\text { Correlaciones subescalas } \\
\text { modelo 2 y EPC }\end{array}$ & $0,631^{\text {a }}$ & $0,683^{\text {a }}$ & $-0,363^{\text {a }}$ \\
\hline
\end{tabular}

EPC: Escala de Preocupación por el Cáncer.

Fiabilidad calculada mediante $\alpha$ de Cronbach.

Rho de Spearman.

a La correlación es significativa al nivel 0,01 (bilateral). 
tras encontrar evidencias de que los ítems 10 y 13 son mejores indicadores del factor Vulnerabilidad que del de Estigma, la fiabilidad para estas subescalas no solo se mantiene alta, sino que aumenta medio punto para Vulnerabilidad respecto a la versión original. Se reportan, asimismo, evidencias a favor de la validez convergente de las 3 subescalas en relación con la EPC.

El número de factores, las relaciones de los ítems con estos, y la relación entre factores están guiados por la evidencia teórica y empírica previa $2,16,17$. El autoconcepto es dinámico, y cambios en él no ocurren con la misma intensidad ni al mismo ritmo en todo el mundo ${ }^{2,11}$. La variabilidad del tiempo transcurrido desde el diagnóstico genético en la muestra, con 4 años de promedio en este estudio, podría explicar el sesgo asimétrico de la distribución muestral de los ítems, con un efecto suelo más marcado en los ítems de Estigma, y un efecto techo en los de Control. Aunque algunos estudios no encuentran efectos del tiempo desde el diagnóstico genético en las dimensiones del autoconcepto ${ }^{15}$, otros describen trayectorias curvilíneas del impacto psicológico del test $B R C A 1 / 2$, siendo el impacto mayor los primeros 6 meses postest ${ }^{27}$. En este estudio los resultados pueden interpretarse en clave de adaptación al CG, y reflejarían la integración (resultado) de ser portador BRCA1/2 en el autoconcepto en términos de baja Estigma, baja Vulnerabilidad $\mathrm{y}$ alto Control en una muestra ya adherida a los seguimientos que ofrecen las unidades de CG. Serían necesarios estudios longitudinales que permitieran conocer el efecto del tiempo en cada uno de los dominios específicos del autoconcepto. Alternativamente, si en posteriores estudios con muestras homogéneas en relación con el tiempo transcurrido desde el diagnóstico genético se siguen observando estos efectos, deberá reconsiderarse la redacción de los ítems, el número de categorías incluidas en la respuesta, o la acotación del número de aspectos incluidos en cada una de las subescalas. En cuanto al modelo puesto a prueba, teniendo en cuenta que para muestras pequeñas se ha establecido un valor de RMSEA de 0,08 como admisible, los índices de ajuste del modelo 1 podrían dar soporte a la estructura original de la escala. No obstante, se ha considerado que el valor del límite superior del intervalo de confianza y la covariancia entre errores de los ítems 4 y 5 mostrada por los IM no permiten dar por válido el modelo. Una posible explicación radica en que para la validación del instrumento original se realizó un ACP que, a diferencia del análisis factorial (AF), no distingue entre variancia común y variancia error, siendo la variancia total la que pretende reproducir. El AF, en contrapartida, se basa en el principio de que las variables tienen error de medida y trata de reproducir tan solo la variancia común. En este sentido, los ítems 4 y 5 son correlativos y empiezan con las mismas palabras, lo que plantea la posibilidad de un efecto de método. Es habitual que los cuestionarios de rendimiento típico contengan ítems redundantes, y en el AF no se puede considerar que los errores entre ítems redundantes sean independientes. Por todo ello se pone a prueba un modelo menos restrictivo ${ }^{20}$.

Por otro lado, la alta correlación entre los factores de Estigma y Vulnerabilidad apunta a una posible designación incorrecta de las relaciones entre los indicadores y los factores latentes, o incluso a que podría tratarse de un mismo factor. Ya en el estudio de desarrollo y validación del instrumento original pueden observarse pesos salientes en más de un factor ${ }^{2}$. Al realizar la prueba de expertos encontramos que los ítems 10 («Pienso mucho en mi prueba genética»)y 13 («Me siento agobiado con esta información»), pertenecientes inicialmente a Estigma, son identificados como indicadores del factor Vulnerabilidad, hallazgo que no contradice la versión original del instrumento, donde estos ítems obtuvieron pesos de 0,36 en dicho factor ${ }^{2}$. Desde un punto de vista teórico ambos ítems tienen un claro componente rumiativo y/o de pensamiento intrusivo propio de la respuesta traumática a episodios vitales estresantes; en este caso, el impacto de ser portador es la pérdida de una identidad como persona $\operatorname{sana}^{4}$, y el alto riesgo a desarrollar $\mathrm{CMOH}$ se erige como una potencial amenaza a la integridad y a la salud propia, incluso la de los familiares, quebrando una de las creencias básicas como es la de invulnerabilidad. En el caso particular del cáncer hereditario, los procesos de atribución que establecen una causa-efecto entre alteración genética y enfermedad podrían instaurar sentimientos de culpa y vergüenza con importantes repercusiones en la identidad, facilitados por un entorno cultural que otorga a los genes un simbolismo de verdadera naturaleza interior y esencia de nuestros ascendentes y descendientes $^{28}$. En el contexto de las enfermedades hereditarias parece difícil desligar un autoconcepto de vulnerabilidad de un autoconcepto de estigma, al atribuir que este mayor riesgo de vulnerabilidad, adoptando el lenguaje propio del CG, se debe a una alteración genética o mutación patogénica, lenguaje que fácilmente puede ser integrado en términos estigmatizadores ${ }^{2}$. En el caso particular del síndrome de Lynch, enfermedad de predisposición hereditaria al cáncer colorrectal, la correspondiente escala de impacto en el autococepto ya contempla las dimensiones de estigma y vulnerabilidad en un solo factor ${ }^{10}$. En nuestro estudio, pruebas previas con 2 factores demostraron que el modelo no se ajusta a un modelo bifactorial, y aun asumiendo el solapamiento entre ambos factores, creemos conveniente mantenerlos diferenciados en tanto que en el $\mathrm{CMOH}$ predicen y están relacionados con necesidades específicas diferentes ${ }^{16,17}$. Además, ambas subescalas obtienen adecuados índices de fiabilidad y una adecuada validez convergente con la EPC. En todo caso, esta alta correlación deberá tenerse en cuenta como potencial fuente de colinealidad en posteriores estudios.

En cuanto a la consistencia interna, la subescala Estigma tiene un $\alpha$ de Cronbach superior a 0,80 en todos los modelos analizados, similar al de la versión original; y Vulnerabilidad, tras incorporar los ítems 10 y 13, aumenta medio punto, hasta 0,843. La fiabilidad de la subescala Control, al igual que ocurre en la versión original, no alcanza el estándar deseado. La definición de la subescala Control propuesta en el instrumento original -autoeficacia, resiliencia y esperanza- pone de relieve su heterogeneidad, que contrasta con el bajo número de ítems que contiene, solo 4, 2 de ellos con pesos factoriales, aunque significativos, bajos. A pesar de estas limitaciones, la prueba de juicio de expertos concluye que los 4 ítems miden Control, y que la versión original del instrumento cuenta con adecuadas medidas de validez convergente, divergente y de criterio $^{2}$. Además, su utilidad ha podido establecerse en estudios empíricos recientes, en los que se ha visto su asociación con bajos niveles de malestar emocional ${ }^{15-17}$. La autoeficacia, la resiliencia y la esperanza desempeñan un rol importante en la adaptación psicológica a hechos potencialmente traumáticos, por lo que, a pesar de la justificada necesidad de completar la fiabilidad y la validez de la subescala, consideramos necesario mantenerla. Alternativamente, se puede complementar la subescala con instrumentos breves aplicados ya en CG, como la percepción personal de control $^{6}$.

La Escala de Autoconcepto en BRCA tiene usos potenciales tanto asistenciales como de investigación. Esta escala permite explorar cómo los impactos específicos del test genético en el autoconcepto se asocian con el bienestar psicológico, la adherencia médica, la toma de decisiones en cirugía profiláctica, o los hábitos de salud, prometedoras líneas a desarrollar que permitirían identificar dianas más específicas en las que focalizar la intervención psicológica, ofreciendo una asistencia clínica más acorde a las necesidades de esta población. Desde un punto de vista asistencial, valorar y detectar si el resultado del test genético está teniendo un impacto positivo o negativo en el autoconcepto tiene claras implicaciones clínicas y permitirá individualizar el proceso de CG respondiendo a las necesidades específicas de cada paciente; al mismo tiempo, también permitirá identificar aquellos pacientes con dificultades de adaptación a su condición de alto riesgo, candidatos a un soporte psicológico más extenso y específico. Así, por ejemplo, en 
portadoras estigmatizadas, trabajar la comunicación con la familia nuclear, especialmente la pareja, puede contribuir a reducir el malestar ${ }^{17}$. La vulnerabilidad, como la preocupación por el cáncer, puede aumentar la ansiedad e interferir con la adherencia médica, mientras que un mayor control y empoderamiento pueden promover conductas de salud ${ }^{2}$, por lo que facilitar un autoconcepto basado en Control puede tener claros beneficios terapéuticos. Precisamente, uno de los atractivos de la escala es que contempla impactos tanto positivos como negativos, siendo las respuestas psicológicas positivas y los beneficios asociados al CG un área de estudio emergente y alentador atendiendo a los resultados prometedores que ya están teniendo en CM intervenciones desde la Psicología Positiva basadas en facilitar el crecimiento postraumático en mujeres con $\mathrm{CM}^{29,30}$.

\section{Financiación}

El presente estudio ha sido posible gracias al soporte del Fondo de Investigación Sanitaria PI10/00748 y PI13/00189, y de la Junta de Barcelona de la Asociación Española Contra el Cáncer. VC ha recibido una beca predoctoral del Instituto de Investigación Biomédica de Bellvitge.

\section{Conflicto de intereses}

Los autores declaran no tener ningún conflicto de intereses.

\section{Agradecimientos}

El equipo investigador quiere agradecer a todo el personal del Programa de Cáncer Hereditario del Institut Català d'Oncologia su colaboración en este trabajo.

\section{Bibliografía}

1. Milne RL, Osorio A, Cajal T, Vega A, Llort G, De la Hoya M, et al. The average cumulative risks of breast and ovarian cancer for carriers of mutations in BRCA1 and BRCA2 attending genetic counseling units in Spain. Clin Cancer Res. 2008;14:2861-9, http://dx.doi.org/10.1158/1078-0432.CCR-07-4436.

2. Esplen MJ, Stuckless N, Hunter J, Liede A, Metcalfe K, Glendon G, et al. The BRCA Self-Concept Scale: A new instrument to measure self-concept in BRCA1/2 mutation carriers. Psychooncology. 2009;18:1216-29.

3. Resta R, Biesecker BB, Bennett RL, Blum S, Estabrooks Hahn S, Strecker $\mathrm{MN}$, et al. A new definition of genetic counseling: National Society of Genetic Counselors' Task Force report. J Genet Couns. 2006;15:77-83, http://dx.doi.org/10.1007/s10897-005-9014-3.

4. Biesecker BB, Erby L. Adaptation to living with a genetic condition or risk: A mini-review. 2008;74:401-7, http://dx.doi.org/10.1111/j.1399-0004. 2008.01088.x.Adaptation.

5. Payne K, Nicholls S, McAllister M, MacLeod R, Donnai D, Davies LM. Outcome measurement in clinical genetics services: A systematic review of validated measures. Value Health. 2008;11:497-508.

6. Kasparian NA, Wakefield CE, Meiser B. Assessment of psychosocial outcomes in genetic counseling research: An overview of available measurement scales. J Genet Couns. 2007;16:693-712, http://dx.doi.org/10.1007/ s10897-007-9111-6.

7. Coyne JC, Benazon NR, Gaba CG, Calzone K, Weber BL. Distress and psychiatric morbidity among women from high-risk breast and ovarian cancer families. J Consult Clin Psychol. 2000;68:864-74.
8. Vos J, van Asperen CJ, Oosterwijk JC, Menko FH, Collee MJ, Garcia EG, et al. The counselees' self-reported request for psychological help in genetic counseling for hereditary breast/ovarian cancer: Not only psychopathology matters. Psychooncology. 2013;22:902-10, http://dx.doi.org/10.1002/pon.3081.

9. Esplen MJ, Stuckless N, Berk T, Butler K, Gallinger S. The FAP self-concept scale (adult form). Fam Cancer. 2009;8:39-50.

10. Esplen MJ, Stuckless N, Gallinger S, Aronson M, Rothenmund H, Semotiuk K, et al. Development and validation of an instrument to measure the impact of genetic testing on self-concept in Lynch syndrome (LS). Clin Genet. 2011:415-23, http://dx.doi.org/10.1111/j.1399-0004.2011.01770.x.

11. Markus H, Wurf E. The dynamic self-concept: A social psychological perspective. Annu Rev Psychol. 1987;38:299-337, http://dx.doi.org/10.1146/ annurev.ps.38.020187.001503.

12. Cantor N, Zirkel S. Personality, cognition, and purposive behavior. En: Pelvin LA, editor. Handbook of personality: Theory and research. New York, NY: Guilford Press; 1990. p. 135-64.

13. Livneh H, Antonak R. Psychosocial adaptation to chronic illness and disability: A primer for counselors. J Couns Dev. 2005;83:12-20, http://dx.doi.org/ 10.1002/j.1556-6678.2005.tb00575.x.

14. Curbow B, Somerfield M, Legro M, Sonnega J. Self-concept and cancer in adults: Theoretical and methodological issues. Soc Sci Med. 1990;31:115-28, http://dx.doi.org/10.1016/0277-9536(90)90053-U.

15. Vodermaier A, Esplen MJ, Maheu C. Can self-esteem, mastery and perceived stigma predict long-term adjustment in women carrying a BRCA1/2mutation? Evidence from a multi-center study. Fam Cancer. 2010;9:305-11, http://dx.doi.org/10.1007/s10689-010-9325-x.

16. Den Heijer M, Seynaeve C, Vanheusden K, Duivenvoorden HJ, Vos J, Bartels C, et al. The contribution of self-esteem and self-concept in psychological distress in women at risk of hereditary breast cancer. Psychooncology. 2011;20:1170-5, http://dx.doi.org/10.1002/pon.1824.

17. Den Heijer M, Vos J, Seynaeve C, Vanheusden K, Kuivenvoorden HJ, TilanusLinthorst $\mathrm{M}$, et al. The impact of social and personal resources on psychological distress in women at risk for hereditary breast cancer. Psychooncology. 2012;21:153-60, http://dx.doi.org/10.1002/pon.1879.

18. Cabrera E, Zabalegui A, Blanco I. Spanish version of the Cancer Worry Scale (CWS). Cross cultural adaptation and validity and reliability analysis. Med Clin (Barc). 2011;136:8-12, http://dx.doi.org/10.1016/j.medcli.2010.04.015 [Spanish].

19. Hay JL, McCaul KD, Magnan RE. Does worry about breast cancer predict screening behaviors? A meta-analysis of the prospective evidence. Prev Med. 2006;42:401-8, http://dx.doi.org/10.1016/j.ypmed.2006.03.002.

20. Brown TA. Confirmatory factor analysis for applied research. New York, NY: The Guilford Press; 2006.

21. Muñiz J, Elosua P, Hambleton RK. Directrices para la traducción y adaptación de los tests: segunda edición. Psicothema. 2013;25:151-7, http://dx.doi. org/10.7334/psicothema2013.24.

22. Graham JW. Missing data analysis: Making it work in the real world. Annu Rev Psychol. 2009;60:549-76, http://dx.doi.org/10.1146/annurev.psych. 58.110405.085530.

23. Byrne BM. Testing for multigroup equivalence of a measuring instrument: $A$ walk through the process. Psicothema. 2008;20:872-82.

24. Jackson DL, Gillaspy JA, Purc-Stephenson R. Reporting practices in confirmatory factor analysis: An overview and some recommendations. Psychol Methods. 2009;14:6-23, http://dx.doi.org/10.1037/a0014694.

25. Nunnally J, Bernstein I. Psychometric theory. 3rd ed New York: McGraw-Hill; 1994.

26. Cohen J. A power primer. Psychol Bull. 1992;112:155-9, http://dx.doi.org/ 10.1037/0033-2909.112.1.155.

27. Beran TM, Stanton AL, Kwan L, Seldon J, Bower JE, Vodermaier A, et al. The trajectory of psychological impact in BRCA1/2 genetic testing: Does time heal? Ann Behav Med. 2008;36:107-16, http://dx.doi.org/10.1007/s12160-008-9060-9.

28. Sumalla EC, Ochoa C, Gil F, Blanco I. Sobre tránsitos, identidades y crecimiento postraumático en cáncer hereditario. Psicooncologia. 2009;6:7-25.

29. Ochoa C, Sumalla EC, Maté J, Castejón V, Rodríguez A, Blanco I, et al. Psicoterapia positiva grupal en cáncer. Hacia una atención psicosocial integral del superviviente de cáncer. Psicooncologia. 2010;7:7-34.

30. Low CA, Bower JE, Kwan L, Seldon J. Benefit finding in response to BRCA1/2 testing. Ann Behav Med. 2008;35:61-9, http://dx.doi.org/10.1007/ s12160-007-9004-9. 